\section{Optimization of HIV-1 infectivity assays}

\author{
Jennifer Jones ${ }^{1}$, William Whitford ${ }^{2}$, Frederic Wagner ${ }^{1}$, and Olaf Kutsch ${ }^{1}$ \\ 1 University of Alabama at Birmingham, Birmingham, AL and ${ }^{2}$ Thermo Fisher \\ Scientific, Logan, UT, USA
}

BioTechniques 43:598-594 (November 2007)

doi $10.2144 / 000112624$

HIV-1 reporter cell lines are the backbone of diagnostic assays, vaccine and drug development efforts. Performing HIV-1 infection experiments in a T cell background is desirable for many reasons. However, a low susceptibility to infection with primary patient isolates in available reporter $T$ cell lines has limited such efforts. We here demonstrate that optimization of HIV-1 receptor expression and the utilization of serum free medium compositions can increase susceptibility of reporter $T$ cell lines to HIV-1 infection by up to two orders of magnitude.
Currently available human immunodeficiency virus-1 (HIV-1) reporter cell lines that are highly susceptible to HIV-1 infection with primary patient isolates are not based on $\mathrm{T}$ cell lines and gain their HIV-1 susceptibility mostly from the presence of surface molecules that nonspecifically trap HIV-1 (1-4). As primary T cells fail to express such molecules, data interpretation from these reporter assays is sometimes complicated. Lacking molecules that can nonspecifically capture HIV-1 particles, T cell-based reporter cell lines generally exhibit a relatively low susceptibility to infection with primary HIV-1 patient isolates, thereby limiting their application. To overcome these limitations, we developed a $\mathrm{T}$ cell-based reporter assay that derives its high susceptibility to infection with primary HIV-1 isolates from optimized HIV-1 receptor expression and from the utilization of a serum-free medium (SFM) composition optimized for this assay.

While most reporter cell lines use luciferase or alkaline phosphatase activity as quantitative read-outs for HIV-1 infection, which produce high signal-over-background ratios but require additional manipulations (5-10), we recently introduced a reporter $\mathrm{T}$ cell line in which HIV-1 infection is directly correlated to enhanced green fluorescence protein (EGFP) expression (JLTRG-R5 cells) (11). These cells hold a stably integrated copy of an HIV-1 long terminal repeat (LTR)-EGFP reporter construct, which is induced through the presence of HIV-1 Tat protein following infection. Infection levels can thus be directly quantified using flow cytometric analysis or plate-based fluorometry. However, JLTRG-R5 cells were designed for high-throughput drug screening (HTS) purposes, and while they can be readily infected with laboratory-adapted HIV-1 clones (e.g., NL43, SG3, BaL), susceptibility to infection with primary HIV-1 patient isolates varies, in particular, as CCR5 expression is low.

To maximize the susceptibility of JLTRG-R5 cells to infection with primary viruses, we initially optimized CD4 and CCR5 expression using retroviral vectors. For this purpose, we cloned the cDNA sequences coding for human CD4 and human CCR5 into a retroviral vector derived from the murine stem cell virus (pMSCVpuro; Clontech Laboratories, Mountain View, CA, USA) using standard PCR techniques. We find that in contrast to other retroviral vectors, gene transfer into human $\mathrm{T}$ cell lines using these murine stem cell virus (MSCV)-based vectors is mostly resistant to DNA methylation and the resulting inactivation of expression during the initial
A

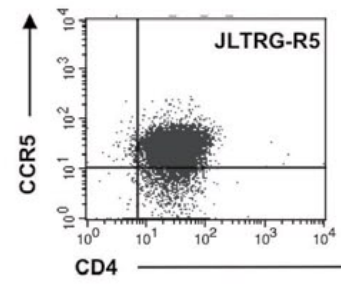

B

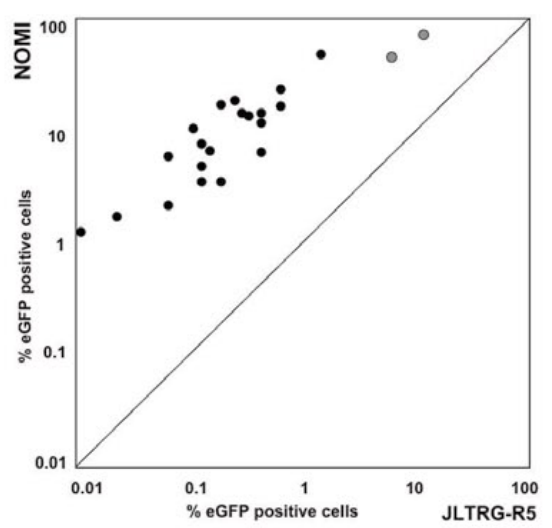

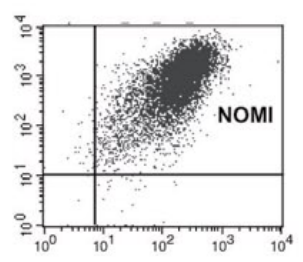
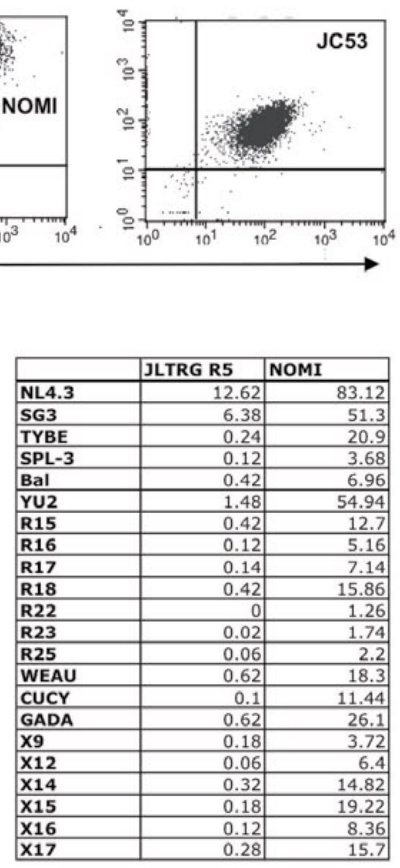

Figure 1. Human immunodeficiency virus-1 (HIV-1) susceptibility of NOMI cells relative to JLTRGR5 cells. (A) CCR5 and CD4 expression on JLTRG-R5, NOMI, and TZM-bl cells, an unrelated HIV-1 reporter cell line. (B) NOMI and JLTRG-R5 cells were infected with $50 \mu \mathrm{L}$ viral supernatants of the indicated viruses derived from peripheral blood mononuclear cell (PBMC) infection cultures (NL43 and SG3 are laboratory adapted $\mathrm{X} 4$ tropic viruses; BaL and YU2 are laboratory adapted R5 tropic viruses; TYBE and SPL-3 are X4 tropic primary patient isolates; R-series viruses are R5 tropic primary patient isolates; $\mathrm{X}$-series viruses are dual tropic primary patient isolates). Infections were performed in the presence of 4 $\mu \mathrm{g} / \mathrm{mL}$ diethylaminoethyl (DEAE)-dextran. On day 2 post-infection, levels of infection in either cell type were determined as the percentage of enhanced green fluorescence protein (EGFP)-positive cells using flow cytometric analysis. For each viral supernatant, infectivity in JLTRG-R5 cells is plotted on the x-axis, while infectivity in NOMI is plotted on the y-axis. Gray circles indicate infection levels for the standard laboratory clones NL43 and SG3 (X4 tropic). 


\section{Benchmarks}
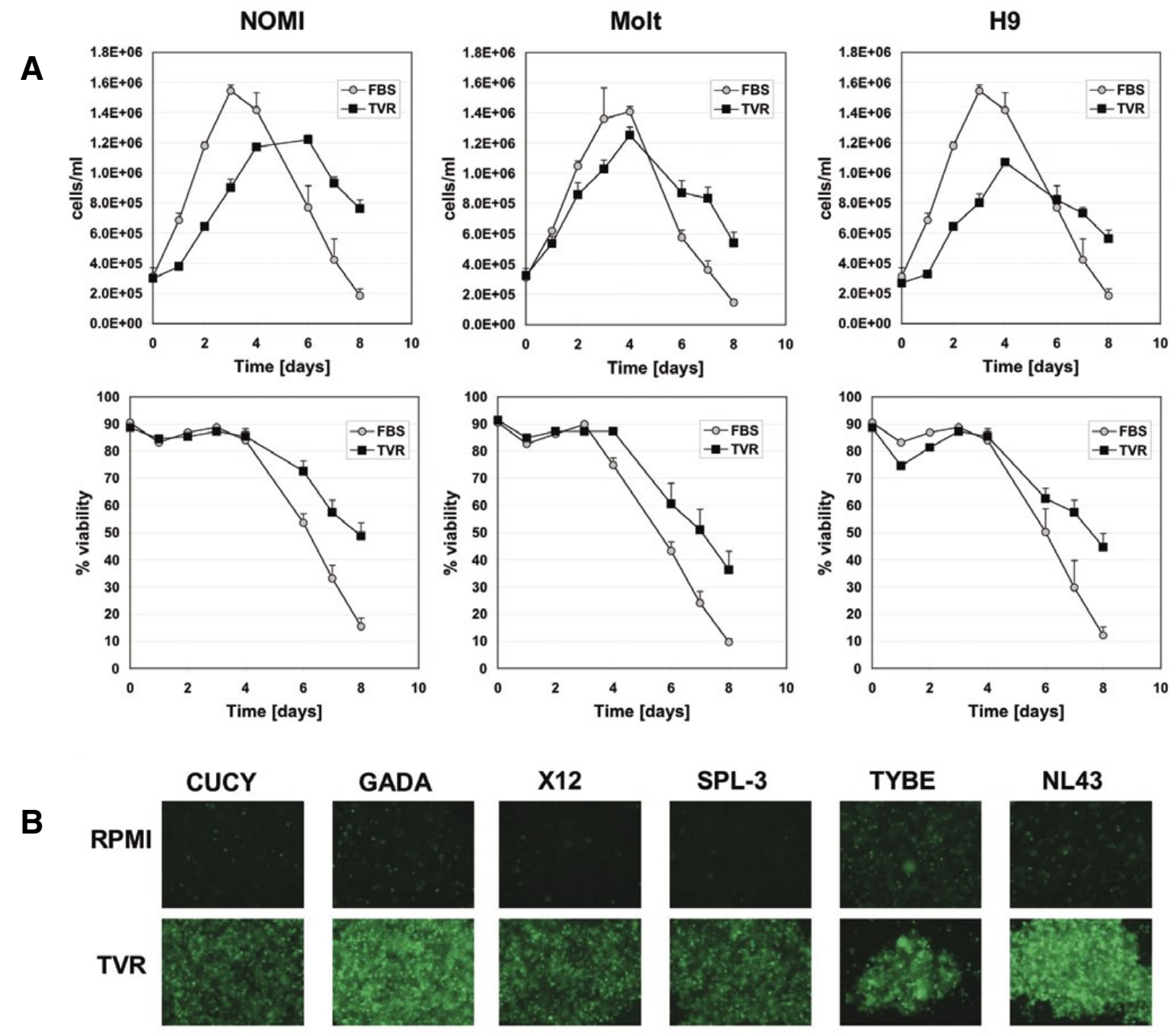

\section{NL43}

C
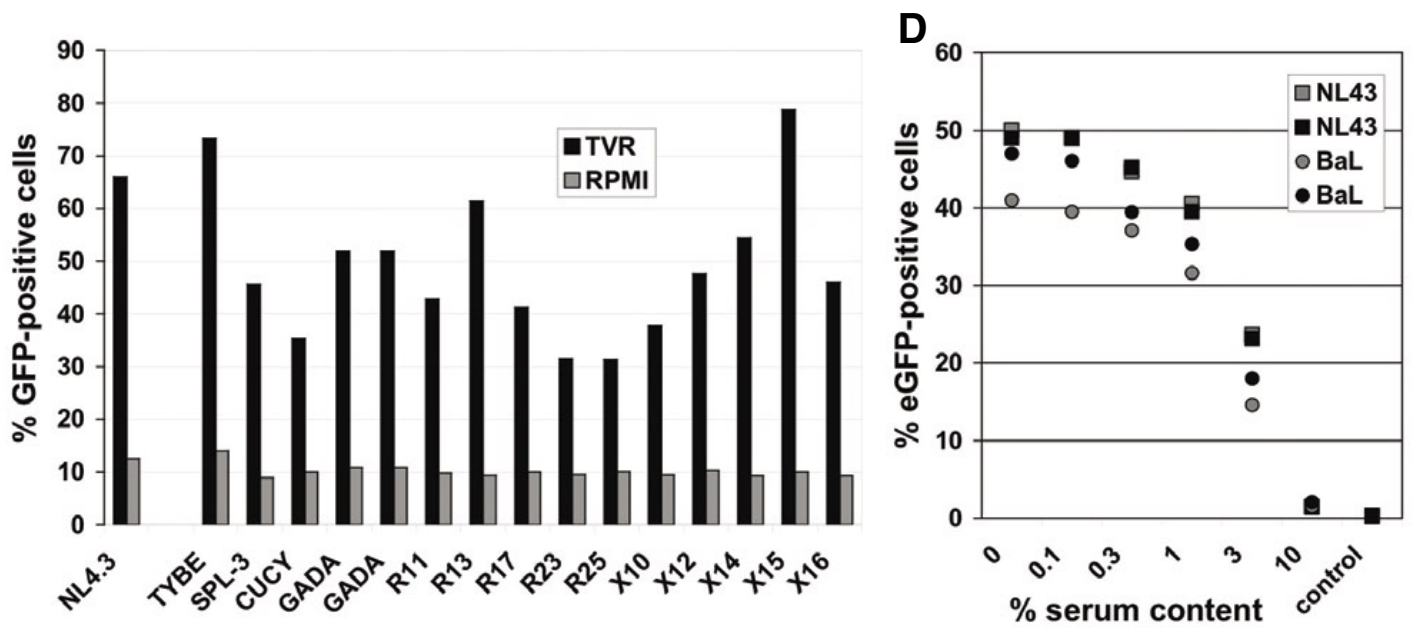

Figure 2. Effects of TVR-23 on human immunodeficiency virus-1 (HIV-1) infection. (A) NOMI, Molt, and H9 cells were seeded at a density of $2 \times 10^{5}$ cells in a total volume of $2 \mathrm{~mL}$ either RPMI supplemented with $10 \%$ fetal bovine serum (FBS) or TVR-23. Cell density and viability at the indicated time points were determined over an 8-day time period using a GUAVA EasyCyte flow cytometer (GUAVA Technologies, Hayward, CA, USA). Results represent the mean \pm SD of three independent experiments. (B) NOMI cells were infected with the indicated viruses and enhanced green fluorescence protein (EGFP) expression was visualized on day 2 post-infection using fluorescent microscopy. All viruses are clinical isolates except NL43. (C) NOMI cells cultured in TVR-23 (black bars) or RPMI/FBS (gray bars) were infected with a panel of HIV-1 viruses, and the percentage of EGFP-positive cells was determined $48 \mathrm{~h}$ post-infection using flow cytometric analysis. (D) Infectious viral supernatants from HIV-1 NL43 (CXCR4-tropic) and BaL (CCR5-tropic) were generated in TVR-23-based cell cultures and FBS was added at the indicated concentrations. Then, NOMI cells were either left uninfected (control) or infected with the serum-spiked viral supernatants, and infection levels were determined after $24 \mathrm{~h}$ using flow cytometric analysis. 


\section{Benchmarks}

transduction. MSCV promoter-driven gene expression is high and very stable in long-term culture, which is a major prerequisite for cell line optimization. JLTRG-R5 cells (Figure 1A) were transduced with the CD4and CCR5-expression vectors and the resulting cell population enriched for high transgene expression using fluorescence-activated cell sorting and finally cloned. The ensuing CD4highCCR5 high reporter $\mathrm{T}$ cell line was termed NOMI cells (Figure 1A) and expressed significantly higher levels of CD4 and CCR5 than JLTRGR5 cells. CD4/CCR5 expression levels are also superior to CD4/ CCR5 expression on TZM-bl (JC53) cells $(3,4)$. TZM-bl (JC53) cells, a frequently used HIV-1 reporter cell line developed from $\mathrm{HeLa}$ cells (a human cervical epithelial carcinoma cell line), derives its high HIV-1 susceptibility primarily from the expression of heparan sulfatebearing adhesion molecules, which are mostly absent on primary $\mathrm{T}$ cells (Reference 2 and data not shown). CD4 and CCR5 expression on NOMI cells were found to be stable for longer than 6 months in continuous culture. As predicted, dependent on the virus clone/isolate, NOMI cells, in comparison to JLTRG-R5 cells, were found to be up to two $\log$ s more susceptible to HIV-1 infection, in particular with CCR5-tropic primary HIV-1 isolates (Figure 1B).

To further improve the assay, we investigated the possibility of utilizing serum-free media (SFM) to enhance HIV-1 infectivity. This strategy is based on previous reports that describe the nonspecific HIV-1 neutralizing capacity of serum proteins, which led to attempts to develop HIV-1 inhibitors based on modified negatively charged serum albumin derivates (12). For this purpose, we sought to adapt NOMI cells to growth in SFM; however, initial attempts to achieve this goal using commercially available SFMs from a panel of vendors failed. We thus embarked on the development of a SFM composition optimized for this assay using plant seed and animal tissue hydrolysates as replacement of some previously serum-supplied nutrients and cofactors. Such formu- lations can produce excellent performance in the absence of serum and can provide a robust support of many cell lines and clones in continuous passage. The proprietary medium composition that resulted from these efforts, coined TVR-23 (Thermo Scientific HyClone, Logan, UT, USA) is a cytokine- and mitogen-free SFM with an unusually complex composition of hydrolysates derived from several plant and animal sources. The TVR-23 formula was developed by optimizing the ratio of soluble fractions from hydrolysates of various plant endosperm and of autolysed Saccharomyces cerevisiae. NOMI cells, as well as several other tested $\mathrm{T}$ cell lines, exhibited growth performance similar to that observed in RPMI supplemented with $10 \%$ fetal bovine serum (FBS) (Figure 2A).

Comparison of HIV-1 susceptibility of NOMI cells cultured in RPMI/FBS or in TVR-23 revealed that infectivity levels in TVR-23 were 3- to 8-fold higher (Figure 2, B and C). Infectious viral supernatants for these experiments were generated by infecting human peripheral blood mononuclear cells (PBMCs) with the respective viruses, the majority of which were obtained from the University of Alabama at Birmingham (UAB) Center for AIDS Research (CFAR) and exhibited CCR5 tropism, with some of the viruses being dualtropic. TYBE and SPL-3 are two $\mathrm{X} 4$-tropic primary viruses and were kindly provided by Dr. Ron Collman (University of Pennsylvania) $(13,14)$. Infection of JLTRG-R5 or NOMI cells was performed in the presence of $4 \mu \mathrm{g} / \mathrm{mL}$ diethylaminoethyl (DEAE)dextrane, a standard procedure to enhance infectivity (www.hiv.lanl. gov/content/nab-reference-strains/ html/Clade-C/TZM-bl_Assay-SOP_ Jan2007.pdf). Most importantly, in some instances, primary viruses that failed to infect NOMI cells in RPMI/ FBS medium readily infected NOMI cells in TVR-23 cultures (e.g., SPL3) (14). Determination of infectious units (I.U.) using NOMI cells and a 1:10 dilution of viral supernatants generated in peripheral mononuclear cells (PBCM)-based infection cultures in RPMI plus 10\% FBS into TVR-23 was found as sensitive as
TZM-bl (JC53) cells infected using a standard protocol (www.hiv.lanl. gov/content/nab-reference-strains/ html/Clade-C/TZM-bl_Assay-SOP_ Jan2007.pdf) and the $\beta$-galactosidase $(\beta$-gal) read-out to determine single infection events (data not shown).

To show that this improved HIV-1 susceptibility is indeed attributable to the absence of serum, we adjusted the infectivity of HIV-1 supernatants (NL43:CXCR4 tropic; BaL:CCR5 tropic) that were produced in TVR23-based cell cultures to generate a $50 \%$ infection and titrated FBS into these preparations. As seen in Figure 2D, addition of FBS concentrations higher than $1 \%$ decreased infection, with $10 \%$ FBS abrogating infection with both viruses, indicating that the superior infectivity seen in TVR23 can be attributed to the absence of serum. A 2- to 3-fold increase in infectivity could also be observed in activated PBMCs from healthy donors infected with an HIV-1 EGFP reporter virus (data not shown) $(15,16)$. However, a delay in the onset of proliferative activity and decreased cell viability in the TVR-23-based PBMC cultures during the initial 3 days following stimulation indicates a requirement of further optimization of TVR-23 for this purpose.

The described two-step optimization process significantly broadens the application range of the previously described JLTRG-R5 cells. The increased viral sensitivity and the broad range susceptibility of NOMI cells to infection with primary HIV-1 isolates should allow for flexible HTS assay design and overcome previous limitations for basic or translational research applications. As infected NOMI cells also produce infectious viral particles, one possible application could be a combination of fluorescence-activated cell sorting for EGFP-positive cells followed by virus propagation in co-cultures with activated primary $\mathrm{T}$ cells to isolate and propagate primary viruses from patient material (data not shown). In addition, TVR-23 could also be used to optimize other HIV-1 reporter cell lines $(15,16)$ or, more general, for HIV-1 replication assays that are based on $\mathrm{T}$ cell cultures. 


\section{Benchmarks}

\section{ACKNOWLEDGMENTS}

This work was supported in part by National Institutes of Health (NIH) grant no. R01AI064012 to O.K. We are grateful to J. Decker for preparing the utilized infectious HIV-1 supernatants. The CXCR4 tropic virus isolates TYBE and SPL-3 were kindly provided by $D r$. Collman (University of Pennsylvania).

\section{COMPETING INTERESTS STATEMENT}

The authors declare no competing interests.

\section{REFERENCES}

1. Platt, E.J., J.P. Durnin, and D. Kabat. 2005. Kinetic factors control efficiencies of cell entry, efficacies of entry inhibitors, and mechanisms of adaptation of human immunodeficiency virus. J. Virol. 79:4347-4356.

2. Mondor, I., S. Ugolini, and Q.J. Sattentau. 1998. Human immunodeficiency virus type 1 attachment to HeLa CD4 cells is CD4 independent and gp120 dependent and requires cell surface heparans. J. Virol. 72:3623-3634.

3. Platt, E.J., K. Wehrly, S.E. Kuhmann, B. Chesebro, and D. Kabat. 1998. Effects of CCR5 and CD4 cell surface concentrations on infections by macrophagetropic isolates of human immunodeficiency virus type 1 . J. Virol. 72:2855-2864.

4. Wei, X., J.M. Decker, H. Liu, Z. Zhang, R.B. Arani, J.M. Kilby, M.S. Saag, X. Wu, et al. 2002. Emergence of resistant human immunodeficiency virus type 1 in patients receiving fusion inhibitor (T-20) monotherapy. Antimicrob. Agents Chemother. 46:1896-1905.

5. Bradley, J., J. Gill, F. Bertelli, S. Letafat, R. Corbau, P. Hayter, P. Harrison, A. Tee, et al. 2004. Development and automation of a 384-well cell fusion assay to identify inhibitors of CCR5/CD4-mediated HIV virus entry. J. Biomol. Screen. 9:516-524.

6. Miyake, H., Y. Iizawa, and M. Baba. 2003. Novel reporter T-cell line highly susceptible to both CCR5- and CXCR4-using human immunodeficiency virus type 1 and its application to drug susceptibility tests. J. Clin. Microbiol. 41:2515-2521.

7. Spenlehauer, C., C.A. Gordon, A. Trkola, and J.P. Moore. 2001. A luciferase-reporter gene-expressing T-cell line facilitates neutralization and drug-sensitivity assays that use either R5 or X4 strains of human immunodeficiency virus type 1. Virology 280:292-300.

8. Daelemans, D., E. De Clercq, and A.M. Vandamme. 2001. A quantitative GFPbased bioassay for the detection of HIV-1 Tat transactivation inhibitors. J. Virol. Methods 96:183-188.
9. Gervaix, A., D. West, L.M. Leoni, D.D. Richman, F. Wong-Staal, and J. Corbeil. 1997. A new reporter cell line to monitor HIV infection and drug susceptibility in vitro. Proc. Natl. Acad. Sci. USA 94:4653-4658.

10. Aguilar-Cordova, E., J. Chinen, L. Donehower, D.E. Lewis, and J.W. Belmont. 1994. A sensitive reporter cell line for HIV-1 tat activity, HIV-1 inhibitors, and T cell activation effects. AIDS Res. Hum. Retroviruses 10:295-301.

11. Ochsenbauer-Jambor, C., J. Jones, M. Heil, K.P. Zammit, and O. Kutsch. 2006. Tcell line for HIV drug screening using EGFP as a quantitative marker of HIV-1 replication. BioTechniques 40:91-100.

12. Jansen, R.W., G. Molema, R. Pauwels, D. Schols, E. De Clercq, and D.K. Meijer. 1991. Potent in vitro anti-human immunodeficiency virus-1 activity of modified human serum albumins. Mol. Pharmacol. 39:818823.

13. Yi, Y., W. Chen, I. Frank, J. Cutilli, A. Singh, L. Starr-Spires, J. Sulcove, D.L. Kolson, et al. 2003. An unusual syncytia-inducing human immunodeficiency virus type 1 primary isolate from the central nervous system that is restricted to CXCR4, replicates efficiently in macrophages, and induces neuronal apoptosis. J. Neurovirol. 9:432-441.

14. Rana, S., G. Besson, D.G. Cook, J. Rucker, R.J. Smyth, Y. Yi, J.D. Turner, H.H. Guo, et al. 1997. Role of CCR5 in infection of primary macrophages and lymphocytes by macrophage-tropic strains of human immunodeficiency virus: resistance to patient-derived and prototype isolates resulting from the delta ccr5 mutation. J. Virol. 71:3219-3227.

15. Kutsch, O., E.N. Benveniste, G.M. Shaw, and D.N. Levy. 2002. Direct and quantitative single-cell analysis of human immunodeficiency virus type 1 reactivation from latency. J. Virol. 76:8776-8786.

16. Levy, D.N., G.M. Aldrovandi, O. Kutsch, and G.M. Shaw. 2004. Dynamics of HIV-1 recombination in its natural target cells. Proc. Natl. Acad. Sci. USA 101:4204-4209.

Received 25 May 2007; accepted 20 September 2007.

Address correspondence to Olaf Kutsch, Department of Medicine, University of Alabama at Birmingham, BBRB 510, 845 19th Street, South Birmingham, AL 35294, USA.e-mail: okutsch@uab.edu

To purchase reprints of this article, contact: Reprints@BioTechniques.com 